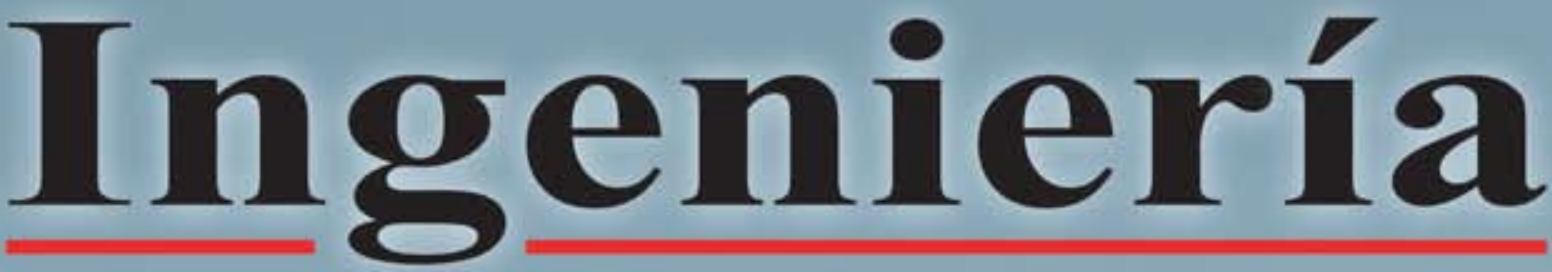

ENERO/DICIEMBRE 2004 - VOLUMEN 14 - N¹ y 2

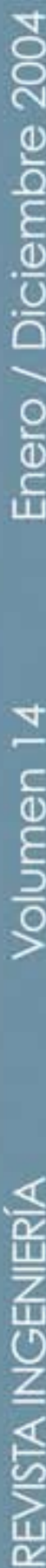
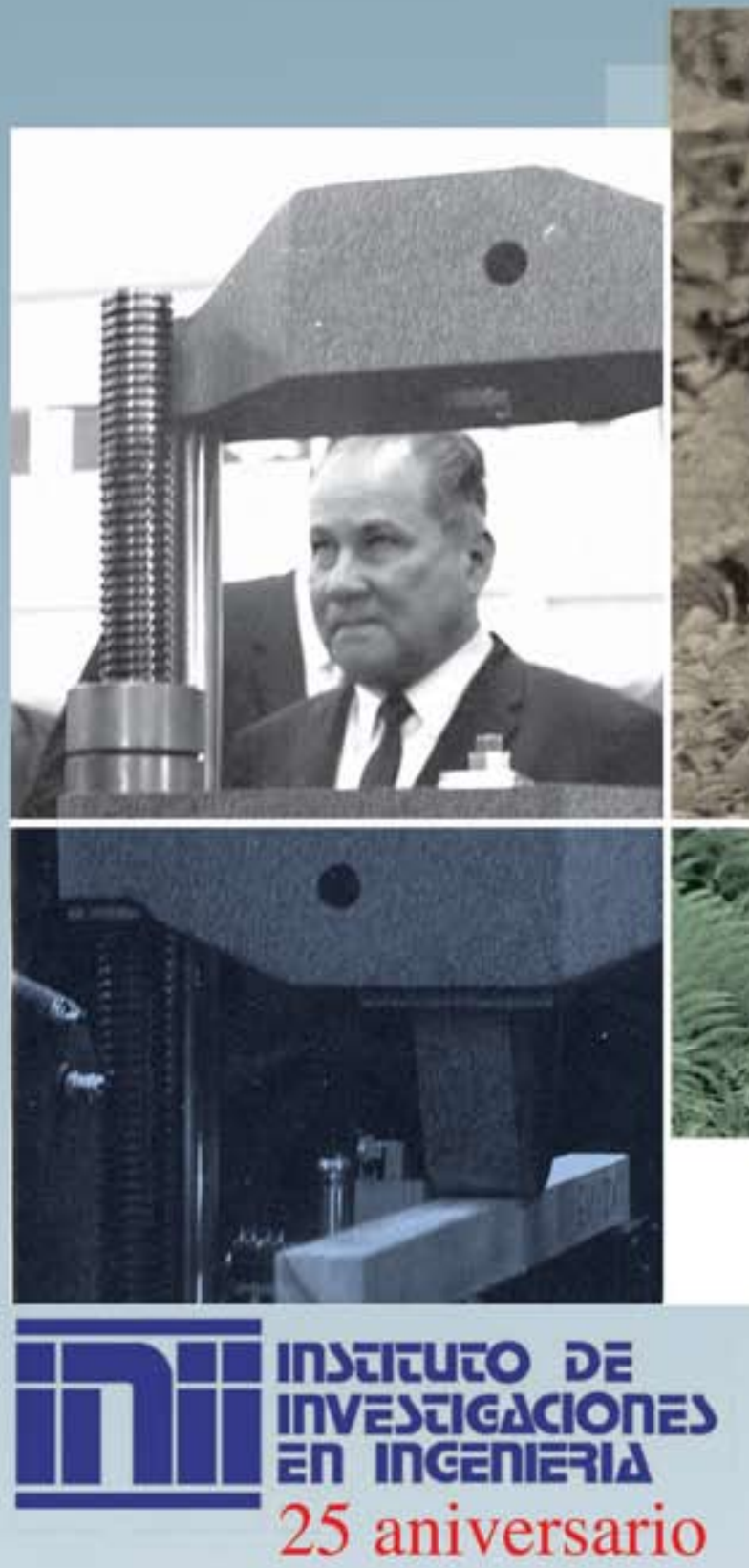

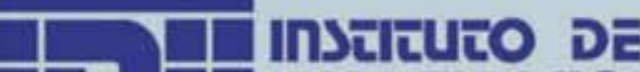
T W Investcscines हn Incenlei|d 25 aniversario






\title{
ANÁLISIS DEL SVC PARA AMORTIGUAR OSCILACIONES DE POTENCIA
}

\author{
Eddie A. Araya Padilla
}

\begin{abstract}
Resumen
La idea fundamental de este trabajo es evaluar el efecto del compensador estático de potencia reactiva o SVC (SVC: Static VAR Compensator) sobre el par de amortiguamiento y el par de sincronización que aporta este dispositivo, a través de un estudio paramétrico. El análisis se desarrolla para un sistema constituido por dos redes regionales vinculadas débilmente a través de una línea de enlace.

Se concluye con este análisis, que el amortiguamiento relativo y el par de amortiguamiento dependen de la potencia nominal del sistema más pequeño, de la impedancia de la línea de enlace y del flujo de potencia transmitido.
\end{abstract}

Palabras clave: compensador estático, enlace débil, amortiguamiento relativo, par de amortiguamiento.

\begin{abstract}
The main purpose of this project is to evaluate the effects produced by the SVC (static VAR compensator) on the damping torque and the relative damping provided by this device, by means of a parametric study. The analysis is developed for a system composed of two regional networks weakly connected by a linking line
\end{abstract}

By this analysis we conclude that the damping torque and the relative damping depend on the nominal power of the smallest system, the line's impedance and the transmitted power.

Keywords: static compensator, linking line, relative damping, damping torque.

Recibido 29-VII-04 • Aceptado 14-X-04

\section{INTRODUCCIÓN}

Las oscilaciones electromecánicas que se encuentran en el ámbito de $0,1 \mathrm{~Hz}$ a $2,5 \mathrm{~Hz}$ son conocidas como oscilaciones de potencia (OP). Si éstas tienen una amplitud creciente y se salen de este límite, provoca que las protecciones del sistema de potencia actúen y que líneas de transmisión y bloques de generación queden fuera de servicio, dividiendo al sistema en islas y con gran probabilidad de que se alcance el colapso de dicho sistema. El amortiguamiento de las oscilaciones es deseable, no solamente porque se reducen las fluctuaciones en las variables controladas, (y por lo tanto, mejora la calidad del servicio eléctrico) sino porque esto significa una mejora en los límites de estabilidad del sistema de transmisión. Este aumento, en régimen permanente, brinda una significativa economía, ya que la expansión de los sistemas de transmisión puede ser pospuesta. Uno de los medios para amortiguar las OP es el SVC.

Las funciones principales de un SVC son, mejorar la estabilidad transitoria y proveer potencia reactiva para el soporte de la tensión en régimen permanente. 
La Figura 1 muestra un esquema de los SVC, el cual está compuesto por reactores controlados por tiristores TCR (TCR: thyristor controlled reactors), capacitores fijos FC (FC: fixed capacitors) y un transformador elevador. En este dispositivo, un control continuo del TCR puede usarse para mejorar el amortiguamiento de las OP.

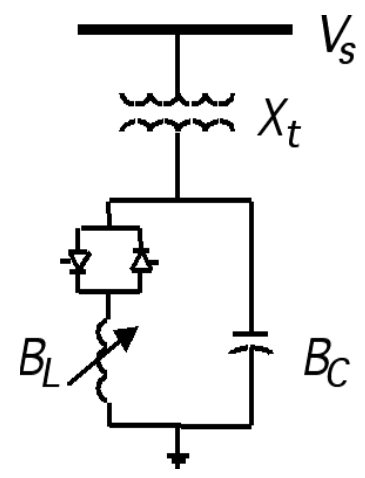

Figura 1. Esquema de un SVC tipo TCR-FC.

Con el SVC en servicio, el límite máximo de transmisión de potencia en régimen permanente, puede aumentarse debido al efecto del ámbito capacitivo del dispositivo. Sin embargo, un control de tensión puro del SVC mejora solamente el par de sincronización y no provee un amortiguamiento apropiado a las OP.

Para amortiguar estas oscilaciones de una manera rápida y efectiva, ciertas variaciones de tensión deben ser permitidas. Esto se logra cuando el SVC es controlado por algunas señales auxiliares, $u_{s}$, suplementarias al lazo de control de tensión, según se muestra en la Figura 2. Estas señales auxiliares deben estar en fase (después de la compensación) con la desviación de velocidad del rotor del generador sincrónico, $\omega_{r \Delta}$, para producir un par de amortiguamiento efectivo. En sistemas reales, $\omega_{r \Delta}$ no puede ser usada como una señal de control, porque usualmente no está disponible en el sitio donde el SVC está instalado, excepto si es derivada en una forma similar a la indicada en (Padiyar \& Varma, 1991) para el caso de una central eléctrica conectada a una barra de potencia infinita.



Figura 2. Diagrama de control del SVC.

\section{EXPRESIÓN ANALÍTICA PARA EL PAR DE AMORTIGUAMIENTO Y EL PERÍODO DE OSCILACIÓN}

Para determinar las acciones correctivas que deben tomarse en cuenta para amortiguar las OP, se deben analizar el amortiguamiento y el período de oscilación de un sistema eléctrico de dos áreas eléctricas constituidas por generadores coherentes, como se muestra en la siguiente Figura 3 (Araya, 1997).

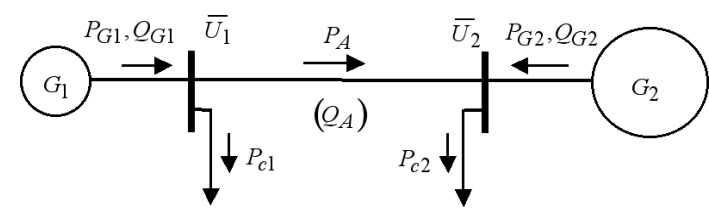

Figura 3. Modelo de dos áreas para OP.

Para este sistema modelo, la ecuación de oscilación del sistema equivalente es dada por:

$$
\begin{aligned}
& \frac{2 H}{\omega_{o}} \frac{d \omega}{d t}+D\left(\omega-\omega_{o}\right)=P_{m}-P_{e} \\
& \delta=\omega-\omega_{o}
\end{aligned}
$$


El intercambio de potencia $P_{A}$ se lleva a cabo a través de la línea de enlace. Se supone que en régimen permanente no hay intercambio de potencia reactiva y que la carga de potencia reactiva de la línea de enlace, corresponde a la potencia reactiva inductiva de los consumidores. La línea de enlace es representada por una reactancia en serie $X_{L}$ y los consumidores, por cargas resistivas.

En la deducción de las expresiones para el amortiguamiento natural $\sigma$ y del período de oscilación $\tau$, se ha despreciado el efecto del regulador de tensión y velocidad de los generadores coherentes.

Analizando la ecuación (3) se observa lo siguiente:

- El riesgo de oscilaciones crecientes aumenta cuando en un sistema en paralelo sale de operación una línea debido a alguna contingencia. Por otro lado, $X_{L}$ aumenta de acuerdo con:

$X_{L}[p u]=X_{L}[\Omega] \frac{S_{T 1 N}[M V A]}{U_{N}^{2}\left[k V^{2}\right]}$

proporcionalmente con la potencia aparente nominal del sistema 1.

- El amortiguamiento disminuye con el aumento de la carga $P_{C 1}$ en el sistema 1 .

- El amortiguamiento aumenta si el sistema 1 está importando energía, $P_{A}<0$. Por el contrario, el amortiguamiento disminuye si el sistema 1 está exportando energía, $P_{A}>0$.

Del mismo modo, de la ecuación (4) se tiene que el período de oscilación:

$\sigma=\left[\frac{D}{H}\right]\left[\frac{X_{G}}{X_{G}+X_{L}}\right]\left[\frac{1}{1+\left(\frac{X_{G} X_{L}}{X_{G}+X_{L}}\right)^{2}\left(\frac{P_{c 1}}{U^{2}}\right)^{2}}\right]\left[C-X_{L} \frac{X_{G} X_{L}}{X_{G}+X_{L}} \frac{P_{A}}{U^{2}} \frac{P_{c 1}}{U^{2}}\right]$

$$
\tau=2 \pi \sqrt{\frac{\left(A^{2}+P_{c 1}^{2}\right) \frac{H}{2 \omega_{o}}}{A\left(A-C \frac{U^{2}}{X_{L}}\right) C-\left[P_{A}^{2}+2 P_{A} P_{c 1}+C P_{c 1}^{2}\right]}}
$$

siendo:

$$
A=\left(\frac{U^{2}}{X_{G}}+\frac{U^{2}}{X_{L}}\right) \quad, \quad C=\sqrt{1-\left(\frac{P_{A} X_{L}}{U^{2}}\right)^{2}}
$$


- Aumenta con la impedancia de la línea $X_{L}$.

- Aumenta con la carga $P_{c l}$.

- Aumenta con la potencia de intercambio $P_{A}$.

- Aumenta con la potencia nominal del sistema 1.

En caso de instalarse un SVC en el punto medio del enlace débil, tal como se muestra en el esquema de la figura 3 ó 4, donde éste tiene el mayor efecto para amortiguar las OP (Padiyar, 1991 y Wang, 1996), la reactancia en serie $X_{L}$ de la línea se modifica a:

$X_{L}=\left(X_{L}-\frac{X_{L}^{2}}{4} B_{S V C}\right)$

De esta forma, dado que las OP se manifiestan como un intercambio de energía eléctrica a través de las líneas de transmisión, otro medio de amortiguarlas es mediante una variación de parámetros en las líneas de transmisión afectadas.

Para comprender el efecto del SVC sobre las OP entre áreas, cada área se modela como una máquina sincrónica y éstas se reducen al equivalente de un generador conectado a una barra de potencia infinita.

\section{MODELO DEL SISTEMA}

Con la aproximación anterior, la inestabilidad del sistema de potencia en régimen permanente se analiza con el modelo de una central eléctrica remota, que envía una gran cantidad de potencia a un sistema principal, a través de una línea de transmisión relativamente débil, como se muestra en la Figura 4.

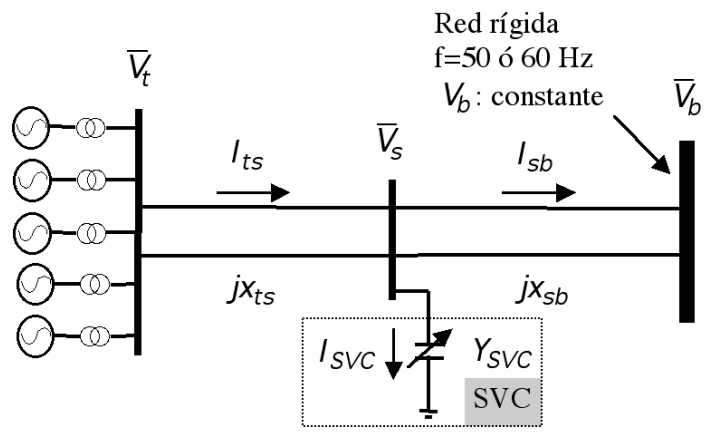

Figura 4. Central eléctrica con SVC en la red.
Esta central eléctrica, a menudo, se representa por una máquina equivalente conectada mediante una impedancia a una barra de potencia infinita para estudios de estabilidad y diseños de sistemas de control. En este modelo, la interacción dinámica entre varias máquinas en la central eléctrica no es considerada, pero es muy adecuada para otros estudios, especialmente cuando las máquinas son idénticas y operan en niveles de carga similares.

Para investigar la contribución del SVC al par de amortiguamiento y sincronización se consideran los siguientes modelos, en su sistema de control:

- Medición de tensión:

$G_{m}(s)=\frac{V_{S V C}}{V_{S}}=\frac{1}{1+s \tau_{m}}$

- Regulador de tensión:

$G_{r}(s)=\frac{K_{r}}{1+s \tau_{r}}$

- Señal estabilizante:

$G_{S}(s)=\frac{\alpha_{2}}{\omega_{r}}$

donde:

$G_{S}(s)=K_{S V C}\left(\frac{s \tau_{W}}{1+s \tau_{W}}\right)\left[\frac{1+s \tau_{1}}{1+s \tau_{2}} \frac{1+s \tau_{3}}{1+s \tau_{4}}\right]\left(\frac{1}{1+s \tau_{5}}\right)\left(\frac{1}{1+s \tau_{6}}\right)$

$H(s)=G_{r}(s) G_{m}(s)$

La señal estabilizante consta de cinco bloques:

- Un filtro de compensación de fase, que consiste de dos etapas, provee el adelanto de fase necesario para compensar el atraso de fase entre la entrada del sistema de excitación y el par eléctrico del generador.

- Un filtro wash-out que determina la frecuencia mínima a partir de la cual el SVC comenzará a aportar amortiguamiento. 
- Una ganancia que delimita la cantidad de amortiguamiento introducido por este dispositivo.

- Dos bloques adicionales que facultan a $\omega_{r}$ como señal auxiliar.

A partir del trabajo de (De Mello \& Concordia, 1969), el modelo de la Figura 5 ha sido tratado por el concepto de pequeña perturbación, sobre las ecuaciones fundamentales de la máquina sincrónica. Las relaciones en el diagrama de bloques se aplican al modelo de dos ejes con un circuito de campo en el eje directo, pero sin los efectos amortiguadores y de saturación. Los parámetros $K_{1} a K_{9}, K_{P}, K_{Q}$ y $K_{V}$ son funciones de las impedancias de la máquina y del sistema, así como del punto de operación del sistema.

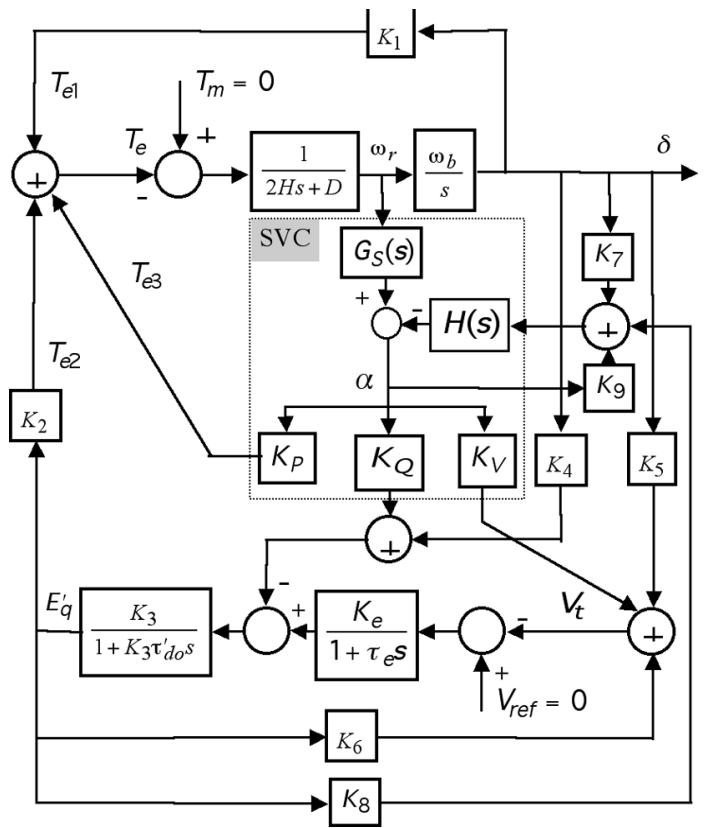

Figura 5. Generador con un SVC en la red eléctrica.

\section{PAR DE SINCRONIZACIÓN Y AMORTIGUAMIENTO}

La evaluación tradicional de los pares de sincronización y de amortiguamiento supone ortogonalidad entre el cambio en el ángulo del rotor y la desviación de velocidad de la máquina. Si una perturbación afecta a la máquina sincrónica, el cambio en el par eléctrico está formado por dos componentes:

$T_{e \Delta}=T_{S} \delta_{\Delta}+T_{D} \omega_{r \Delta}$

donde:

- $T_{S} \delta_{\Delta}$ es el componente del par eléctrico en fase con la desviación en el ángulo del rotor $\delta_{\Delta}$ y se designa como par de sincronización; $T_{S}$ es el coeficiente del par de sincronización.

- $T_{D} \omega_{r \Delta}$ es el componente del par eléctrico en fase con la desviación de velocidad del rotor $\omega_{r \Delta}$ y se designa como par de amortiguamiento; $T_{D}$ es el coeficiente del par de amortiguamiento.

Si se utiliza la relación $\omega_{r \Delta}=\mathrm{s \delta}_{\Delta}$, la ecuación. (12) toma la forma:

$T_{e \Lambda}=\left(T_{S}+j \omega T_{D}\right) \delta_{\Lambda}=T_{C} \boldsymbol{\delta}_{\Lambda}$

De modo que el par eléctrico puede expresarse como un número complejo, donde la parte real es el par de sincronización, $T_{S}$ y la parte imaginaria es el par de amortiguamiento, $\omega T_{D}$. Por lo tanto, los coeficientes del par de sincronización y amortiguamiento son definidos por:

$T_{S}=\operatorname{Re}\left\{T_{C}\right\}$

$T_{D}=\frac{1}{\omega} \operatorname{Im}\left\{T_{C}\right\}$

Para propósitos de este análisis se aplica la metodología anterior al modelo de la Figura 5 para obtener las figuras 6, 7,8 y 9 bajo diferentes condiciones de operación. Por otro lado, se muestran las expresiones analíticas de $T_{S}$ y $T_{D}$ suponiendo las siguientes aproximaciones: 
- El efecto de $K_{Q}$ y $K_{v}$ sobre el par de amortiguamiento es despreciable según (Wang, 1996).

- La constante $K_{6}$ tiene una magnitud mucho mayor que los otros parámetros.

- Sólo se consideran frecuencia de oscilación menores a $1 \mathrm{~Hz}$.

Con base en lo anterior, las expresiones de $T_{S}$ y $T_{D}$ para el modelo de la Figura 5 son:

$T_{S} \cong K_{1}-\frac{K_{2} K_{5}}{K_{6}}+\frac{K_{P} K_{r}}{K_{6}} \frac{\left(K_{5} K_{8}-K_{6} K_{7}\right)}{\left(1+K_{r} K_{9}\right)}$

$T_{D} \cong \frac{K_{2} K_{5}}{K_{6}^{2} K_{e}}\left[\left(\tau_{d o}^{\prime}+\frac{\tau_{e}}{K_{3}}\right)-\frac{K_{4} K_{6}}{K_{5}} \tau_{e}\right]-\frac{K_{P} K_{E}}{\left(1+K_{r} K_{9}\right)}$

donde:

$K_{E}=\frac{K_{r}}{K_{6}}\left[\frac{\left(\tau_{r}+\tau_{m}\right)}{\left(1+K_{r} K_{9}\right)}\left(K_{5} K_{8}-K_{6} K_{7}\right)+\frac{K_{5} K_{8}}{K_{6} K_{e}}\left(\tau_{d o}^{\prime}+\frac{\tau_{e}}{K_{3}}\right)\right]$

Como puede verse, en régimen permanente el aporte del SVC a los coeficientes $T_{S}$ y $T_{D}$ es despreciable, puesto que ningún parámetro de la señal estabilizante $G_{S}(s)$ está presente. Los términos adicionales en las ecuaciones (16) y (17) corresponden únicamente al lazo de control de tensión del SVC. La eficacia del SVC puede modificarse en alguna medida a través del cambio de signo de la constante $K_{5}$ según lo observado en (De Mello \& Concordia, 1969).

\section{PRUEBA DE EFECTIVIDAD DEL SVC.}

Para mostrar la efectividad del SVC, se presenta el comportamiento de $T_{S}$ y $T_{D}$ para diferentes condiciones de operación. Los datos correspondientes al sistema de excitación, la máquina sincrónica y la reactancia de la línea de transmisión son tomados de (De Mello \&
Concordia, 1969). El modelo del SVC es el mismo utilizado por (Wang \& Swift, 1996). Los lazos del control de amortiguamiento y tensión son similares a los que se indican en (Padiyar \& Varma, 1991).

La Figura 6 muestra el coeficiente $T_{D}$ para diferentes condiciones de operación, cuando el vínculo de transmisión está compuesto por una o dos líneas y el SVC está fuera de servicio. En el caso de dos líneas $T_{D}$ es pequeño pero positivo y disminuye al aumentar la potencia reactiva suministrada por el generador, hasta llegar a ser negativo cuando $P$ se aproxima a $0,93 p u(Q=0,2$ $p u)$. En caso de que el sistema pierda una línea debido a una falla, $T_{D}$ disminuye marcadamente. $\mathrm{Si}$ el sistema es estable después del transitorio, pero presenta $T_{S}>0$ y $T_{D}>0$, las OP tendrán una amplitud creciente causando inestabilidad oscilatoria al sistema.

Las Figuras 7, 8 y 9 presentan el efecto del lazo de control de amortiguamiento del SVC.

En la Figura 7, el coeficiente de sincronización $T_{S}$ varía con la frecuencia de oscilación. El límite en régimen permanente se alcanza cuando la potencia transmitida $P$ se aproxima a 0,7 $p u$ ( $\mathrm{f}=0,6 \mathrm{~Hz}$ ). No obstante, el coeficiente de amortiguamiento $T_{D}$ presenta un aumento notable a partir de $\mathrm{P}=0,25 \mathrm{pu}$ según la frecuencia de oscilación.

La Figura 8 muestra el efecto de considerar los lazos de control de tensión y amortiguamiento del SVC. En este caso $T_{S}$ disminuye al aumentar la frecuencia delimitando el límite de potencia en régimen permanente por encima de $0,95 p u$ ( $\mathrm{f}=1,0 \mathrm{~Hz}$ ). No obstante, $T_{D}$ es positivo, pero de magnitud inferior, si se le compara al caso anterior de la Figura 7.

La Figura 9 indica la efectividad de $T_{S}$ y $T_{D}$ para distintas condiciones de operación cuando el SVC trabaja con los lazos de control de tensión y amortiguamiento. Un incremento en la potencia reactiva entregada por el generador aumenta $T_{S}$, y por tanto, su límite en régimen permanente, mientras que el efecto sobre $T_{D}$ es similar al caso de la Figura 8. 


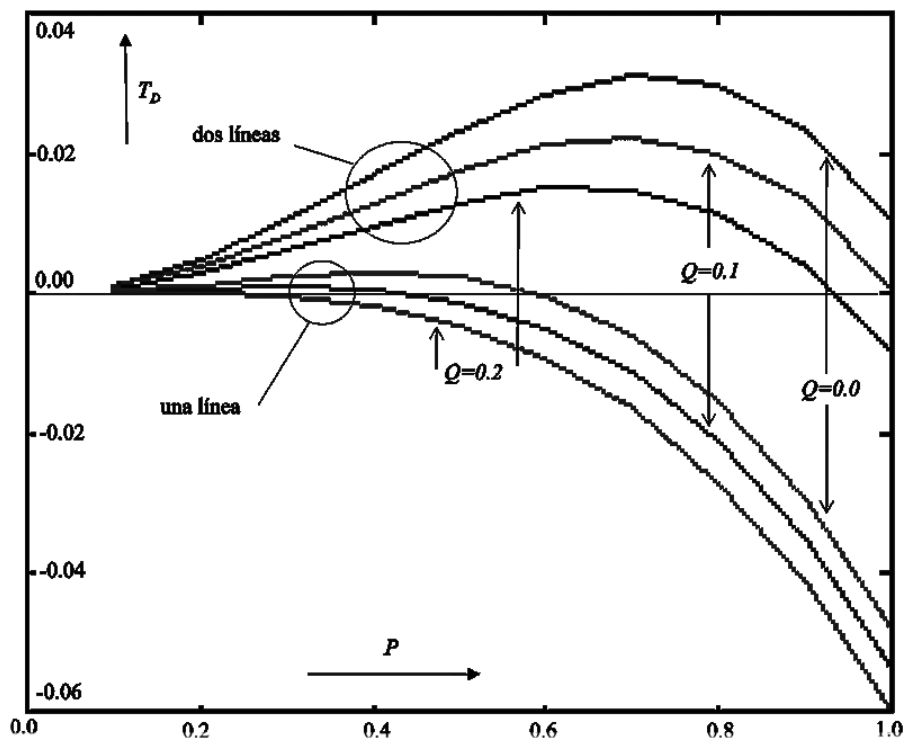

Figura 6. $T_{D}$ vs potencia activa en $p u$. Potencia reactiva como parámetro en $p u$. El SVC está fuera de operación.

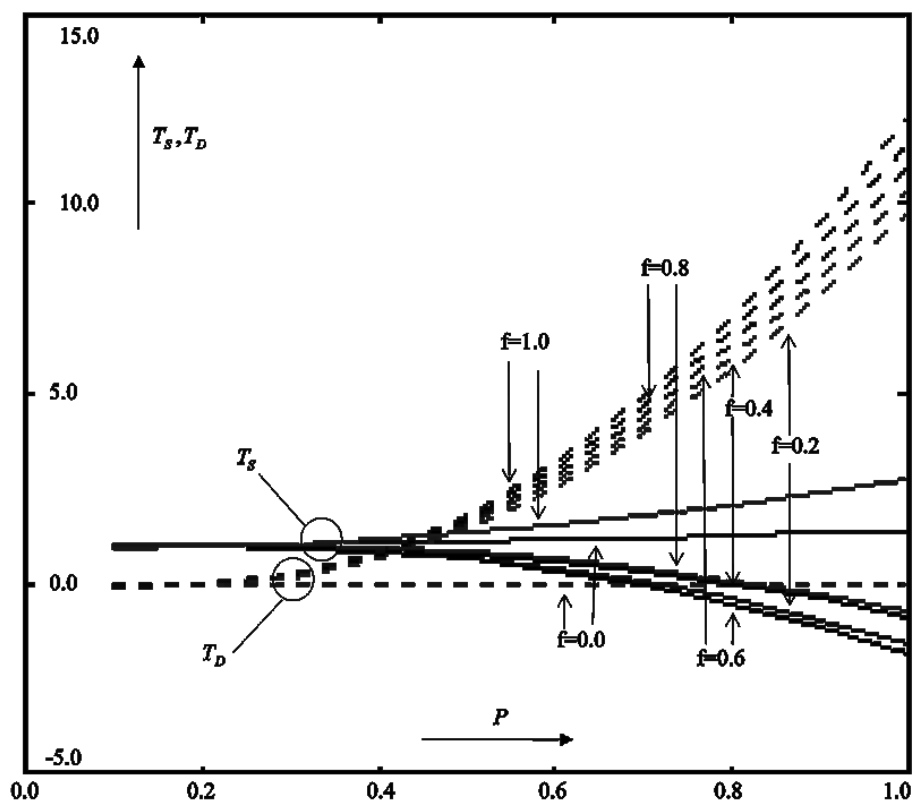

Figura 9. $T_{S}$ y $T_{D}$ vs potencia activa en $p u$, para varias frecuencias de oscilación $\mathrm{Hz}$, con una potencia reactiva nula. El SVC está en operación, $\alpha=108^{\circ}$. 




Figura 8. $T_{S}$ y $T_{D}$ vs potencia activa en $p u$ transportada a través de una línea de transmisión, para varias frecuencias de oscilación en $\mathrm{Hz}$, con una potencia reactiva nula. El SVC está en operación, $\alpha=108^{\circ}$.



Figura 9. $T_{S}$ y $T_{D}$ vs potencia activa en pu, para varias potencias reactivas, con una frecuencia de oscilación de 1,0 Hz. El SVC está en operación, $\alpha=108^{\circ}$. 


\section{CONCLUSIONES}

En vista del análisis anterior, las conclusiones son:

- Cuando se instala el SVC, el coeficiente del par de sincronización aumenta ligeramente a muy bajas frecuencias.

- El coeficiente del par de amortiguamiento aumenta con el incremento en la potencia transmitida.

- En presencia del SVC, la potencia reactiva suministrada por el generador tiene un efecto favorable sobre el coeficiente del par de sincronización, pero ligeramente perjudicial sobre el coeficiente del par de amortiguamiento.

- La frecuencia de oscilación tiene mayor impacto sobre $T_{S}$ que sobre $T_{D}$.

\section{SIMBOLOGÍA}

D: $\quad$ coeficiente de amortiguamiento

$H$ : $\quad$ constante de inercia

$s=j \omega: \quad$ operador de Laplace

$T_{e}: \quad$ par eléctrico

$T_{m}$ : par mecánico

$T_{S} \Delta \delta$ : cambio en el par sincronizante

$T_{D} \cdot \Delta \omega: \quad$ cambio en el par de amortiguamiento

$\delta$ : $\quad$ ángulo entre el eje en cuadratura y la barra de potencia infinita

$\omega: \quad$ velocidad angular del rotor del generador, alrededor del punto de operación

$\Delta: \quad$ subíndice que indica un pequeño cambio en alguna variable

\section{APÉNDICE}

La simbología utilizada se encuentran descrita principalmente por (De Mello \& Concordia,

1969), excepto las constantes, y que se indican a continuación.

$$
\begin{gathered}
K_{7}=\left.\frac{\Delta V_{S}}{\Delta \delta}\right|_{E_{q}^{\prime}, \alpha} \\
K_{8}=\left.\frac{\Delta V_{S}}{\Delta E_{q}^{\prime}}\right|_{\delta, \alpha}
\end{gathered}
$$$$
K_{9}=\left.\frac{\Delta V_{S}}{\Delta \alpha}\right|_{\delta, E_{a}^{\prime}}
$$

Los valores de los parámetros del SVC, utilizados en la simulación, son:

Ganancia del SVC:

$K_{s v c}=50,0$.

Constante de tiempo del circuito de medición: $\tau_{m}=0,02 \mathrm{~s}$.

Constante de tiempo del regulador de tensión $\tau_{r}=0,05 \mathrm{~s}$.

Ganancia del regulador de tensión:

$K r=50,0$.

\section{BIBLIOGRAFÍA}

Araya, E. (1997, noviembre). Efectividad del SVC ante diferentes condiciones de carga. En: III Congreso Latinoamericano de Generación y Transporte de Energía Eléctrica. São Paulo. Brasil.

De Mello F. P. \& Concordia, C. (1969). Concepts of synchronous machine stability as affected by excitation control. IEEE Transaction on Power Apparatus and Systems, 4(PAS88),316-327. 
Martins, N. \& Lima,L.T.G.(1990). Determination of suitable locations for power system stabilizers and static VAR compensators for damping electromechanical oscillations in large power systems. IEEE Transaction on Power Systems, 4(PWRS-5), 1455-1469.

Padiyar, K. R. \& Varma, R. K. (1991). Damping torque analysis static VAR system controllers. IEEE Transaction on Power Systems, 2(PWRS-6),458-465.

Wang, F. \& Swift, F. J. (1996). Application of the Phillips-Heffron Model in the analysis of the damping torque contribution to power systems by SVC damping control. Electrical Power \& Energy Systems, 5(18),307-313
Zhou, E. Z. (1993). Application of static VAR compensators to increase power system damping. IEEE Transaction on Power Systems, 2(PWRS-8),655-661.

\section{SOBRE EL AUTOR}

\section{Eddie A. Araya Padilla}

Ingeniero electricista, Ph, D.; profesor del Departamento de Sistemas de Potencia de la Escuela de Ingeniería Eléctrica de la Universidad de Costa Rica

Teléfonos: 207-4329 ó 207-5146

Facsímil: 207-4139

Correo electrónico: earaya@eie.ucr.ac.cr 